\title{
Prognostic role of ALK-1 and h-TERT expression in glioblastoma multiforme: correlation with ALK gene alterations
}

\author{
Dalia Elsers ${ }^{1}$, Doaa F. Temerik ${ }^{2}$, Alia M. Attia ${ }^{3}$, A. Hadia ${ }^{4}$, Marwa T. Hussien $^{5}$ \\ 'Department of Pathology, Faculty of Medicine, Assiut University, Assiut; Departments of ${ }^{2}$ Clinical Pathology, ${ }^{3}$ Radiation Oncology, ${ }^{4}$ Medical Oncology, and \\ ${ }^{5}$ Oncologic Pathology, South Egypt Cancer Institute, Assiut University, Assiut, Egypt
}

\begin{abstract}
Background: Anaplastic lymphoma kinase (ALK) is a receptor tyrosine kinase that is expressed in the developing central and peripheral nervous systems during embryogenesis. Human telomerase reverse transcriptase ( $h$-TERT) protein resumption is the main process of preservation of telomeres that maintains DNA integrity. The present study aims to evaluate the prognostic role of ALK-1 and h-TERT protein expression and their correlation with ALK gene alterations in glioblastoma multiforme (GBM). Methods: The current study is a retrospective study on a cohort of patients with GBM $(n=53)$ that attempted to detect $A L K$ gene alterations using fluorescence in situ hybridization. ALK-1 and h-TERT proteins were evaluated using immunohistochemistry. Results: Score 3 ALK-1 expression was significantly associated with male sex, tumor multiplicity, Ki labeling index (Ki LI), and type of therapeutic modality. Score 3 h-TERT expression exhibited a significant association with Ki LI. ALK gene amplifications (ALK-A) were significantly associated with increased Ki LI and therapeutic modalities. Score 3 ALK-1 protein expression, score $3 \mathrm{~h}$-TERT protein expression, and ALK-A were associated with poor overall survival (OS) and progression-free survival (PFS). Multivariate analysis for OS revealed that ALK gene alterations were an independent prognostic factor for OS and PFS. Conclusions: High protein expression of both ALK-1 and h-TERT, as well as ALK-A had a poor impact on the prognosis of GBM. Further studies are needed to establish the underlying mechanisms.
\end{abstract}

Key Words: ALK-1; h-TERT; ALK gene; Glioblastoma multiforme; Prognosis

Received: February 1, 2021 Revised: March 14, 2021 Accepted: March 15, 2021 Corresponding Author: Marwa T. Hussien, MD, PhD, Department of Oncologic Pathology, South Egypt Cancer Institute, Assiut University, Assiut 171516, Egypt Tel: +20-88-208671, Fax: +20-088-2087709, E-mail: marwat.hussien@aun.edu.eg

Glioblastoma multiforme (GBM) is a grade IV glioma and is considered the most common and lethal malignant central nervous system (CNS) neoplasm in adults. The incidence rate of GBM is three cases per 100,000 persons in the adult population, with a median survival of 15 months. CNS tumor diagnoses should consist of a histopathological name based on genetic features [1]. According to the recent World Health Organization classification of CNS tumors, GBM that lacks the isocitrate dehydrogenase (IDH) mutation is termed GBM, IDH-wild type, while tumors that harbor an IDH mutation are termed GBM, IDH-mutant type. Tumors that lack any diagnostic mutation are categorized as GBM, not otherwise specified [2]. GBM necessitates robust diagnostic and management strategies [1].

The anaplastic lymphoma kinase (ALK) is a member of the insulin receptor superfamily of receptor tyrosine kinases. The genomic locus that codes the ALK gene is located at human chro- mosome 2p23. The ALK protein has restricted expression in some tissues such as pericytes, endothelial, and neural cells in the brain, and is also found in some types of lymphoma, lung carcinoma, neuroblastomas, and GBMs [3]. Some studies showed that negative ALK-1 expression in GBM predicted better overall survival (OS) [4]. Other studies revealed no significant impact on OS with different ALK expression levels in GBM [5].

$A L K$ gene rearrangements are mostly linked to subcellular changes in the ALK protein, which can assessed by immunohistochemistry (IHC) and can likewise be evaluated with the fluorescence in situ hybridization (FISH) technique with higher sensitivity and specificity [5]. Both tests use an in vitro diagnostic assay that the Food and Drug Administration (FDA) approved for crizotinib response prediction. FISH is a qualitative test that detects $A L K$ gene rearrangements with all potential fusion partners. Other methods of molecular testing such as real-time poly- 
merase chain reaction targeted only the fusion site. Therefore, other probable clinically important fusion sites will not be detected. ALK gene analysis using FISH helps to detect a specific type of gene aberration, such as gene rearrangement and amplifications [6]. ALK gene amplification (ALK-A) has been recognized in several types of cancer as anaplastic large cell lymphoma (ALCL), hepatocellular carcinoma (HCC), esophageal squamous cell carcinoma, and GBM [7].

Telomerase is composed of a reverse transcriptase catalytic subunit human telomerase reverse transcriptase (h-TERT) and an RNA template, the human telomerase RNA component (hTERC). The h-TERT coded by the TERT gene is an active catalytic protein subset located at chromosome 5p15.33. The other subset of telomere is hTERC or hTR, coded by the human telomerase RNA component (TERC gene), which positioned at chromosome $3 \mathrm{q} 26$. In cancer cells, the resumption of h-TERT protein is the chief process of telomere preservation [8]. The expression of h-TERT is an important determinant of telomere activity. Generally, h-TERT is not expressed in normal tissues, which has been attributed to strict h-TERT regulation. However, h-TERT was highly expressed in malignant tumors including breast cancer, HCC, thyroid cancer and gliomas [9]. Some studies on GBM patients documented that patients with absent h-TERT expression had significantly longer OS than patients with strong h-TERT expression [10]. However, another study revealed no significant effect of h-TERT expression on survival [11].

The standard treatment for newly diagnosed GBM is maximum safe resection followed by concurrent temozolomide (TMZ) and radiotherapy with adjuvant TMZ for six cycles. This study reported a median OS of 14.6 months after median follow-up duration of 28 months [12]. Despite multimodal treatment of GBM, patient outcomes are still non-satisfying for neuro-oncologists. Molecular characterization of GBM with clinical concern is still under research for emerging targeted therapy. A novel targeted ALK inhibitor therapy as crizotinib, which is an FDAapproved treatment for lung cancer, is currently used in clinical trials for ALK-positive ALCL and in a few GBM patients [13]. However, emerging resistance to treatment with ALK inhibitors in patients remains a major concern. A recent study revealed that a subset of patients with ALK-positive lung adenocarcinomas harbor additional TERT amplification, which leads to unstable genomes with obvious fast relapse and therapeutic failure [14].

To date, there is no data detailing the relationship between ALK-1 and h-TERT expression in GBM. The present study aimed to assess ALK-1 and h-TERT protein expression, their correlation with $A L K$ gene alterations in GBM prognosis, and their relationship to conventional therapy for GBM.

\section{MATERIALS AND METHODS}

This was a retrospective study that included 53 patients primarily diagnosed with GBM. All were recruited and diagnosed in Pathology Department, Assiut University Hospital and South Egypt Cancer Institute, between April 2014 and April 2018. Patients were followed until May 2020. All patients were eligible at age 20-80 years, if they had no previous diagnosis of cancer and no previous CNS surgery for any cause. The exclusion criteria were patients with primary malignant brain tumors other than GBM or with brain metastasis, and patients with no follow-up records.

With regards to the treatment modalities used in the current research, all patients underwent surgical intervention, which included gross total resection, subtotal resection, or biopsy. Following surgical intervention, fractionated conformal radiation therapy was delivered to Gross tumor volume 1, which included the T2/FLAIR abnormality and the surgical cavity if present for a total dose of $46 \mathrm{~Gy}$ in 23 fractions at 2 Gy per fraction, once daily, for five days per week. Gross tumor volume 2 included T1 contrast enhanced abnormality and the surgical cavity if present for a boost dose of $14 \mathrm{~Gy}$ in seven fractions with 2 Gy per fraction, once daily, for five days per week. All patients were treated using a megavoltage linear accelerator and photon energies of $6 \mathrm{MV}$ or more. Adjuvant radiotherapy only was given in 12 patients (22.6\%) while the remaining patients received chemotherapy as a part of a treatment protocol. Chemotherapy treatment consisted of TMZ, which was given concomitantly with radiotherapy $\left(75 \mathrm{mg} / \mathrm{m}^{2} /\right.$ day, started from the first day of radiotherapy until the end of radiation) in 27 patients $(50.9 \%$ ) or given as concurrent chemoradiotherapy (CCRT) and adjuvant (150-200 mg/m²/for 5 days/every 28 days for 6 or 12 cycles) in 14 patients (26.4\%).

Follow-up evaluation included history and neurological examination, laboratory investigations, assessment of treatment related toxicity, and magnetic resonance imaging (MRI) or magnetic resonance spectroscopy imaging that were available for review. $\mathrm{Pa}$ tients were evaluated for response using MRI and or magnetic resonance spectroscopy, which were performed within 48 hours of surgery, before the first cycle, after every 3 cycles of adjuvant TMZ, and every three months after termination of treatment.

The GBM specimens used for evaluation of ALK-1, h-TERT immunohistochemical protein expression, and $A L K$ gene alterations using the FISH technique. Clinicopathological parameters collected from the patient's archives sheets included patient age, 
sex, tumor site, presence of tumor calcification, tumor multiplicity, tumor size, and Ki-67 labeling index (Ki LI) with cutoff point of $14 \%$ [15].

\section{Immunohistochemistry}

Formalin-fixed paraffin-embedded (FFPE) slides from the GBM tissue blocks, were retrieved from pathology lab and included for IHC study. The slides stained with hematoxylin and eosin were reviewed histologically before staining by the two pathologic consultants in this research. The FFPE blocks were cut into $3-4-\mu m$ thickness, and then put on positively charged glass slides (PCS). Sections were de-paraffinized and rehydrated, followed by antigen retrieval, which was done with Tris-EDTA in a water bath at $90^{\circ} \mathrm{C}$ for 45 minutes. The primary monoclonal mouse anti-Human ALK/CD246 antibody (clone ALK-1), ready-to-use (code IR641, 117498-002, CVR No. 33211317, Dako, Glostrup, Denmark), Ki-67antibody (clone MIB-1), ready-to-use (code IR626, primary mononclonal mouse, Dako), and a primary rabbit polyclonal anti-Human TERT antibody (catalog \#213737, United State Biological 4 Technology, Salem, MA, USA) were applied. Anti-TERT antibody was used at a dilution of 1/75 (optimum dilution according to datasheet). Both incubated for one hour at room temperature in an airtight humid chamber. A universal staining kit "Ultra Vision Detection System Anti-Polyvalent, HRP/DAB (ready-to-use)" (catalog \#TP-015-HD, LAB VISION Corp., Fremont, CA, USA) was applied following the manufacturer's instructions.

\section{Evaluation of ALK-1 and $\mathrm{h}$-TERT IHC expression}

ALK-1 positivity was identified as a brown cytoplasmic expression. A four-tier scoring system was used for evaluation of ALK-1 positivity [5]; score 1 was corresponded to weak cytoplasmic expression of ALK-1, moderate cytoplasmic expression was considered as score 2, and strong cytoplasmic expression was considered as score 3 (Fig. 1). Negative staining was scored as 0 .

Positive brown nuclear staining of h-TERT was deemed positive. A three-tier evaluation system was used for scoring of hTERT IHC protein expression [11]. Score 1 corresponded to nuclear expression in $<5 \%$ of tumor cells. h-TERT expression in between 5 and $50 \%$ tumor cells was scored as 2, and expression in more than $50 \%$ of tumor cells was deemed to score 3 (Fig. 2). Positive cytoplasmic staining of ALK-1 protein in anaplastic lymphoma cells was used as a positive control. Positive nuclear staining of h-TERT in melanoma cells were used as positive control. Negative control done using the same protocol of IHC unless the addition of the primary antibody on tissue section of specific positive controls.

Ki-67 positivity was identified as a brown nuclear expression. $\mathrm{KI}$ LI was defined as the percentage of positive tumor nuclei in 1,000 tumor cells with cutoff value of $14 \%$ [15].

\section{Fluorescence in situ hybridization}

An ALK break-apart probe set (XT ALK BA Dual Color, Break Apart Rearrangement Probe [reference number: D-6001-100OG], Metasystems, Altlussheim, Germany) was used for FISH to detect gene rearrangements and copy number changes. In each case, we examined around 200 nuclei from at least 5-8 areas. We excluded nuclei with apparent overlapping or truncation.

Four-micrometer-thick tissue sections were cut from FFPE GBM tissue and were put on PCS. The unstained slides were placed overnight at $60^{\circ} \mathrm{C}$ on a hotplate. Then, the slides were immersed 3 times in xylene for 5 minutes and dehydrated twice in $100 \%$ ethanol for 5 minutes at room temperature. In sequence, the slides were immersed for 20 minutes in $0.2 \mathrm{~N} \mathrm{HCl}$, in purified water for 3 minutes, and in $1 \mathrm{M}$ sodium thiocyanate at $80^{\circ} \mathrm{C}$ for 30 minutes. The slides were incubated for 30 minutes in Protease Solution previously warmed to $37^{\circ} \mathrm{C}$ after removal of excess water and washed in purified water for 3 minutes. Then, dehydration of slides in $70 \%, 80 \%$, and $100 \%$ ethanol for one minute each was done and slides were allowed to dry. Then, they were placed in a dark room. Ten microliters of probe mixture were applied to a slide, immediately covered by a coverslip, and sealed with rubber cement. They were placed in a hybridizer instrument (DakoCytomation, Glostrup, Denmark) at $73^{\circ} \mathrm{C}$ for 3 minutes followed by an overnight hybridization at $37^{\circ} \mathrm{C}$. At the end of the hybridization period, we removed the rubber cement from the slides and placed them in $2 \times$ saline sodium citrate (SSC; post hybridization wash) at $73^{\circ} \mathrm{C}$ temperature for 2 minutes, then immersed them for 1 minute in $2 \times$ SSC at room temperature and allowed the slides to dry. Ten microliters of DAPI counterstain was applied to the target area and covered by a coverslip [6].

\section{Analysis of $A L K$ gene alterations using FISH technique}

We analyzed the prepared slides under an oil immersion objective (100X) with a fluorescence microscope (M1, Carl Zeiss Microscopy GmbH, Gottingen, Germany) equipped with appropriate filters and a charge-coupled device camera using FISH imaging with the capturing software Metafer 5 (a Metafer slide scanning system [Metasystems]). Non-rearranged ALK showed fusion (yellow signals) or very close abutment of the probes adjacent to the $3^{\prime}$ (red) and the $5^{\prime}$ (green) ends of the gene. Rearranged ALK appeared as splitting of $3^{\prime}$ and $5^{\prime}$ signals. Tumor tissues 

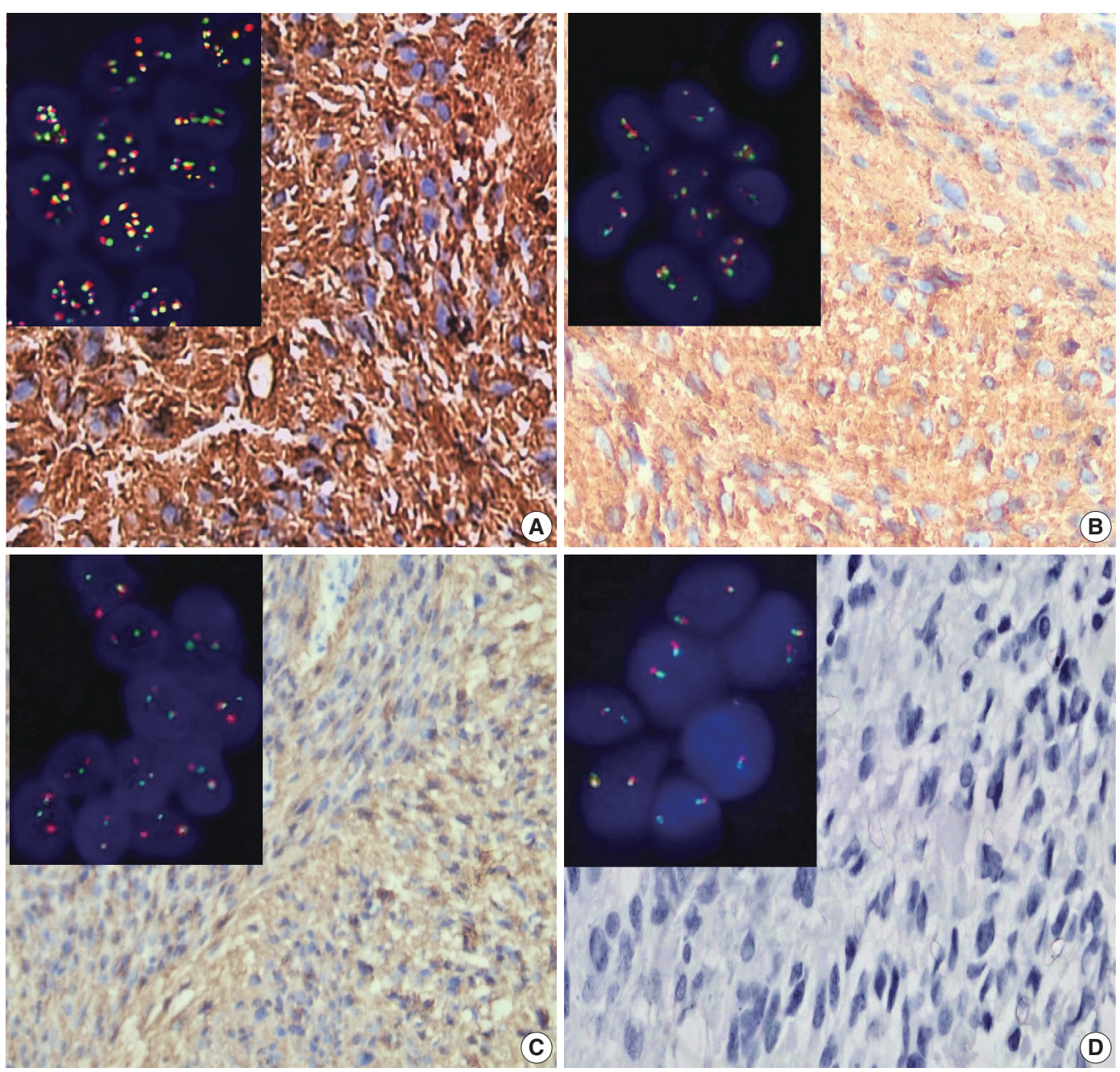

Fig. 1. Expression of anaplastic lymphoma kinase 1 (ALK-1) immunohistochemistry in tumor cells and ALK gene alterations in glioblastoma multiforme (GBM). (A) A case of GBM shows strong cytoplasmic expression of ALK-1 in tumor cells (score 3). The inset illustrates ALK gene amplification for the same case. (B) A case of GBM shows moderate cytoplasmic staining of ALK-1 in tumor cells. The inset illustrates ALK gene gain for the same case. (C) A case of GBM shows weak cytoplasmic expression of ALK-1 in tumor cells (score 1). The inset illustrates ALK gene rearrangement for the same case. (D) A case of GBM showed negative expression of ALK-1 (score 0). The inset illustrates that the $A L K$ gene was negative for rearrangement with a normal copy number for the same case.

were considered ALK-FISH positive ( $A L K$-rearranged) if $>15 \%$ tumor cells showed splitting of red and green signals [16]. The mean cutoff copy number of 3 to 5 fusion signals in $\geq 10 \%$ of cells represented $A L K$-copy number gain (ALK-CNG), while the presence of $\geq 6$ copies of ALK per cell in $\geq 10 \%$ of analyzed cells represented ALK-A (Fig. 1) [17].

\section{Statistical analysis}

The analysis for this study was done through using the SPSS ver. 21 (IBM Corp., Armonk, NY, USA). Fisher exact test was used to detect the association between ALK protein, TERT protein expression, $A L K$ gene alterations, and various clinicopatho- logical data. Chi-square test was used only when $\leq 20 \%$ of the cells had an expected count less than 5. Correlation between $A L K$ gene alterations and ALK and TERT IHC protein expression were done via Spearman correlation coefficient test. OS was calculated from the date of surgical resection to the date of death from any cause or last follow-up. Progression-free survival (PFS) was calculated from the date of surgical resection to the date of progression or date of last follow-up or death.

Kaplan-Meier curves were used to analyze OS and PFS. Comparison of survival was determined by log-rank test. Multivariate analysis using Cox proportional hazard model of predictors of outcome variables were applied. The value of significance was 

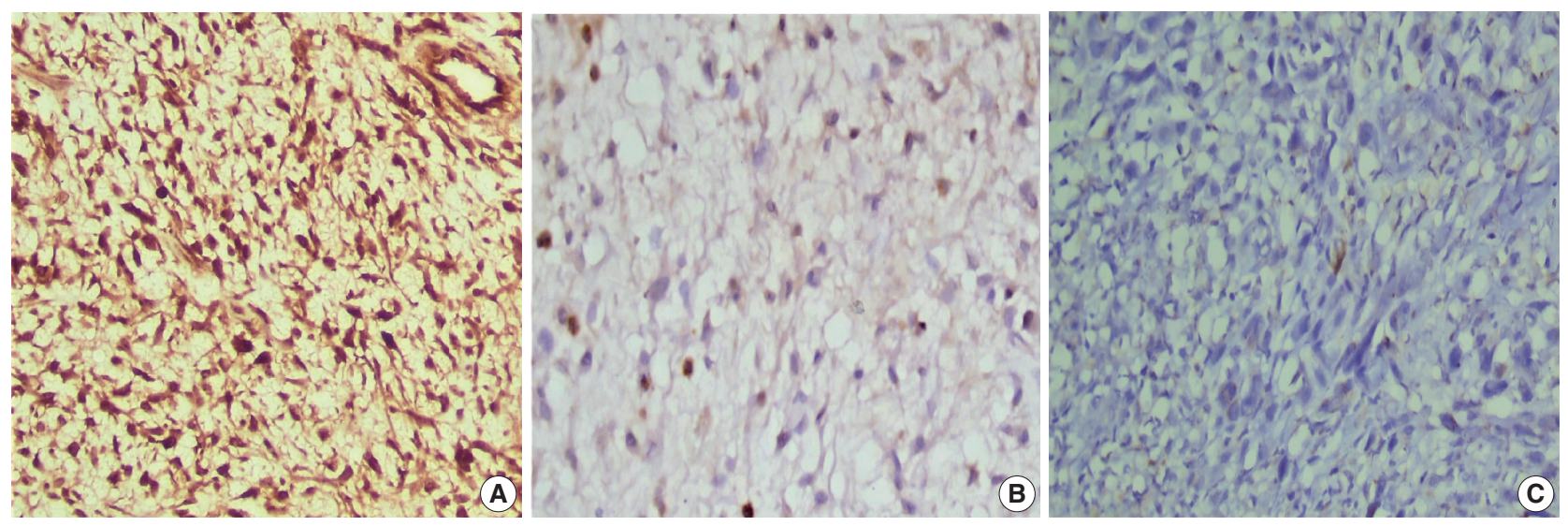

Fig. 2. Expression of human telomerase reverse transcriptase (TERT) immunohistochemistry in tumor cells of glioblastoma multiforme. (A) Strong nuclear expression of TERT in $>50 \%$ of tumor cells (score 3). (B) Moderate nuclear staining of TERT in 5\%-50\% of tumor cells (score 2). (C) Weak nuclear expression of TERT in $<5 \%$ of tumor cells (score 1 ).

determined as $\mathrm{p}<.05$.

\section{RESULTS}

The current study included 53 GBM patients. The study included 17 patients (32.1\%) who were $<50$ years of age, while 36 patients $(67.9 \%)$ were $\geq 50$ years old. Thirty-six cases $(67.9 \%)$ were males. The most prominent tumor location was at the parieto-occipital region, constituting $28.4 \%$ of patients. The median tumor size was $5 \mathrm{~cm}$. Calcification was present in 32 cases $(60.4 \%)$. There were multiple tumors in six cases (11.3\%).

After a median follow-up duration of 12 months (range, 3 to 19 months), 21 out of 53 patients (39.6\%) were still alive (Table 1).

\section{Association between ALK-1 IHC protein expression and clinicopathological parameters}

ALK-1 expression was detected in 45 cases out of 53 (84.9\%). Strong ALK cytoplasmic expression (score 3) was noted in 17 GBM tumors (32.1\%). Thirteen cases (24.5\%) showed moderate cytoplasmic expression (score 2), while 15 cases (28.3\%) showed weak cytoplasmic expression (score 1). Eight cases (15.1\%) were negative (score 0 ).

Score 3 ALK-1 expression showed significant association with male sex ( $\mathrm{p}=.038$ ), tumor multiplicity ( $\mathrm{p}=.046)$, and Ki LI ( $\mathrm{p} \leq$ .001 ). The type of therapeutic modality was positively associated with ALK-1 protein expression ( $\mathrm{p}=.030$ ). The clinicopathological associations with ALK-1 protein IHC expression are summarized in Table 2.

\section{Association between h-TERT IHC protein expression and clinicopathological parameters}

h-TERT expression was detected in the studied cases with variable percentages and staining intensities. Strong h-TERT nuclear expression in $>50 \%$ of tumor cells (score 3) was noted in 29 $(54.7 \%)$ cases, while moderate expression in $5 \%-50 \%$ of tumor cells (score 2) was present in 16 cases (30.2\%) and eight cases $(15.1 \%)$ showed weak expression in $<5 \%$ of tumor cells (score 1 ).

h-TERT expression showed significant association with the presence of calcifications $(\mathrm{p}=.016)$. Score $3 \mathrm{~h}$-TERT expression exhibited a significant association with Ki LI ( $\mathrm{p}=.005)$. There was no significant correlation between h-TERT expression and other clinicopathological variables (Table 2).

\section{Association between ALK gene alterations and clinicopathological parameters}

Thirty-four cases out of 53 showed $A L K$ gene alterations (64.2\%). ALK-A was detected in four cases (7.5\%), and all of these cases had strong ALK immunohistochemical expression. ALK-CNG was noted in 12 cases (22.6\%), while 18 cases (34.0\%) showed $A L K$ gene rearrangement. Nineteen cases (35.9\%) were negative. ALK-A was significantly associated with increased Ki LI ( $\mathrm{p}=$ $.044)$. The type of therapeutic modality was positively associated with ALK gene alterations $(\mathrm{p}=.027)$. There was no significant association between ALK gene alteration and clinicopathological variables (Table 3).

\section{Correlation between ALK gene alterations, ALK-1 and h-TERT IHC protein expression}

A strong positive correlation was noted between ALK protein IHC expression and $A L K$ gene alterations $(\mathrm{p}=.001, \mathrm{r}=0.616)$. 
Table 1. Clinicopathological characteristics of the patients

\begin{tabular}{|c|c|}
\hline Variable & No. (\%) \\
\hline \multicolumn{2}{|l|}{ Age (yr) } \\
\hline$<50$ & $17(32.1)$ \\
\hline$\geq 50$ & $36(67.9)$ \\
\hline \multicolumn{2}{|l|}{ Sex } \\
\hline Male & $36(67.9)$ \\
\hline Female & $17(32.1)$ \\
\hline \multicolumn{2}{|l|}{ Site } \\
\hline $\mathrm{CC}$ & $4(7.5)$ \\
\hline $\mathrm{FP}$ & $12(22.6)$ \\
\hline PO & $15(28.4)$ \\
\hline TO & $7(13.2)$ \\
\hline PS & $6(11.3)$ \\
\hline $\mathrm{TP}$ & $9(17.0)$ \\
\hline \multicolumn{2}{|l|}{ Calcification } \\
\hline Absent & $21(39.6)$ \\
\hline Present & $32(60.4)$ \\
\hline \multicolumn{2}{|l|}{ Multiplicity } \\
\hline Single & $47(88.7)$ \\
\hline Multiple & $6(11.3)$ \\
\hline \multicolumn{2}{|l|}{ Tumor size } \\
\hline Median (interquartile range) & $5(4-7)$ \\
\hline \multicolumn{2}{|l|}{ Type of surgical resection } \\
\hline GTR & $19(35.0)$ \\
\hline STR & $25(47.0)$ \\
\hline Biopsy & $9(17.0)$ \\
\hline \multicolumn{2}{|l|}{ Ki LI (\%) } \\
\hline$<14$ & $37(69.8)$ \\
\hline$\geq 14$ & $16(30.2)$ \\
\hline \multicolumn{2}{|l|}{ Status } \\
\hline Living & $21(39.6)$ \\
\hline Dead & $32(60.4)$ \\
\hline \multicolumn{2}{|l|}{ Therapeutic modalities } \\
\hline RTH only & $12(22.6)$ \\
\hline CCRT & $14(26.4)$ \\
\hline CCRT and adjuvant TMZ & $27(50.9)$ \\
\hline
\end{tabular}

CC, corpus callosum; FP, fronto-parietal; PO, parieto-occipital; TO, tempero-occipital; PS, parasagittal; TP, tempero-parietal; GTR, gross total resection; STR, subtotal resection; Ki LI, Ki labeling index; RTH, radiotherapy; CCRT, concurrent chemoradiotherapy; TMZ, temozolomide.

Moderate correlation was noted between $A L K$ gene alterations and TERT IHC ( $\mathrm{p}=.007, \mathrm{r}=0.476$ ), as (score 2) TERT expression was associated with $A L K$ gene rearrangement $(56.2 \%)$, while (score 3) TERT expression was associated with ALK-CNG and ALK-A (51.8\%). There was a strong positive correlation between ALK-1 and TERT IHC protein expression ( $\mathrm{p}=.002, \mathrm{r}=0.602)$. The relationships and correlations between $A L K$ gene alterations, ALK, and TERT IHC protein expression are presented in Tables 3 and 4, respectively.

\section{Outcome analysis}

The median follow-up duration of the 53 GBM patients was 12 months (range, 3 to 19 months). During follow-up, 32/53 patients (60.4\%) died as a result of tumor progression. According to Kaplan-Meier analysis, the median OS was 12 months (95\% confidence interval [CI], 10.449 to 13.551), while the 19-month OS rate was $39.6 \%$. A total of 36/53 patients (67.9\%) developed disease progression. The median time to progression was 10 months (range, 2 to 19 months). According to Kaplan-Meier analysis, the median PFS was 10 months $(95 \% \mathrm{CI}, 7.860$ to 12.140). The PFS rate at 19 months was $32.1 \%$.

ALK-1 protein expression (score 3), h-TERT protein expression (score 3), and ALK-A were associated with poor OS and PFS ( $\mathrm{p}<.001, \mathrm{p}=.031$, and $\mathrm{p}<.001)$ and $(\mathrm{p}<.001, \mathrm{p}=.040$, and $\mathrm{p}<.001)$, respectively. Cases that exhibited high Ki LI had poor OS and short PFS ( $\mathrm{p}=.016)$ and $(\mathrm{p}=.022)$, respectively (Figs. 3, 4).

Regarding the type of therapeutic modalities, patients treated with adjuvant radiotherapy only had poor OS and PFS compared to those who were treated with CCRT or CCRT and adjuvant $\operatorname{TMZ}(\mathrm{p}=.002)$ and $(\mathrm{p}=.004)$, respectively (Table 5 , Figs. 3E, 4E).

Multivariate analysis for OS and disease-free survival was applied to clinicopathological features that were significant in univariate analysis to adjust for confounders. Our results revealed that ALK gene alteration was the only independent prognostic factor for OS and PFS ( $\mathrm{p}<.001$; hazard ratio [HR], 7.514; $95 \%$ CI, 3.292 to 17.155 ) and (p $\leq .001 ; \mathrm{HR}, 4.711 ; 95 \%$ CI, 2.429 to 9.136), respectively (Table 6).

\section{DISCUSSION}

GBM exhibits a vast group of modifications, both genetic and epigenetic, which create a great number of mutation subsets, some of which have a proven effect in survival and therapy response [1].

In the present study, high ALK-1 expression showed a significant association with male sex, however, no significant relation between ALK-1 expression and patient's age. These findings are not matched with the study done by Karagkounis et al. [5], which reported that ALK overexpression is more common in older individuals ( $>59$ years) and that was no association between ALK expression and patient's sex. This discrepancy was due to division of their cases into subgroups according to IDH1 protein expression with cutoff median age of 59 years, which was not implemented in the current study.

One persistent debate is whether ALK-1 immunohistochemical overexpression was associated with $A L K$ gene mutation or amplification in GBM cases. In the current study, we reported that 
Table 2. Association between ALK-1, h-TERT IHC protein expression, and clinicopathological parameters

\begin{tabular}{|c|c|c|c|c|c|c|c|c|c|}
\hline \multirow{2}{*}{ Parameter } & \multicolumn{5}{|c|}{ ALK-1 IHC } & \multicolumn{4}{|c|}{ h-TERT IHC } \\
\hline & Score 0 & Score 1 & Score 2 & Score 3 & $p$-value & Score 1 & Score 2 & Score 3 & $p$-value \\
\hline Age (yr) & & & & & .236 & & & & $.123^{\mathrm{a}}$ \\
\hline$<50$ & $2(11.8)$ & $6(35.3)$ & $6(35.3)$ & $3(17.6)$ & & $3(17.6)$ & $8(47.1)$ & $6(35.3)$ & \\
\hline$\geq 50$ & $6(16.7)$ & $9(25.0)$ & 7 (19.4) & 14 (38.9) & & $5(13.9)$ & $8(22.2)$ & 23 (63.9) & \\
\hline Sex & & & & & .038 & & & & $.267^{a}$ \\
\hline Male & $7(19.4)$ & $6(16.7)$ & $10(27.8)$ & $13(36.1)$ & & $6(16.7)$ & $13(36.1)$ & $17(47.2)$ & \\
\hline Female & $1(5.9)$ & $9(52.9)$ & 3 (17.6) & 4 (23.5) & & $2(11.8)$ & $3(17.6)$ & $12(70.6)$ & \\
\hline Site & & & & & .425 & & & & .119 \\
\hline $\mathrm{CC}$ & $2(50.0)$ & $1(25.0)$ & 0 & $1(25.0)$ & & 0 & $3(75.0)$ & $1(25.0)$ & \\
\hline $\mathrm{FP}$ & $1(8.4)$ & $4(33.3)$ & 4 (33.3) & $3(25.0)$ & & $2(16.7)$ & $2(16.7)$ & $8(66.6)$ & \\
\hline PO & $2(13.3)$ & $7(46.7)$ & $2(13.3)$ & $4(26.7)$ & & $5(33.3)$ & $2(13.3)$ & $8(53.4)$ & \\
\hline TO & $2(28.5)$ & $1(14.3)$ & $3(42.9)$ & $1(14.3)$ & & $1(14.3)$ & $4(57.1)$ & $2(28.6)$ & \\
\hline PS & 0 & 0 & $2(33.3)$ & $4(66.7)$ & & 0 & $1(16.6)$ & $5(83.4)$ & \\
\hline $\mathrm{TP}$ & $1(11.2)$ & 2 (22.2) & $2(22.2)$ & $4(44.4)$ & & 0 & $4(44.4)$ & $5(55.6)$ & \\
\hline Calcification & & & & & .315 & & & & $.016^{\star}$ \\
\hline Absent & $1(4.8)$ & $6(28.6)$ & $5(23.8)$ & $9(42.8)$ & & $3(14.3)$ & $2(9.5)$ & 16 (76.2) & \\
\hline Present & $7(21.9)$ & $9(28.1)$ & $8(25.0)$ & $8(25.0)$ & & $5(15.6)$ & $14(43.8)$ & $13(40.6)$ & \\
\hline Multiplicity & & & & & .046 & & & & .501 \\
\hline Single & $8(17.1)$ & $14(29.8)$ & $13(27.7)$ & $12(25.5)$ & & $8(17.0)$ & 15 (31.9) & 24 (51.1) & \\
\hline Multiple & 0 & $1(16.7)$ & 0 & 5 (83.3) & & 0 & $1(16.7)$ & 5 (83.3) & \\
\hline Tumor size & & & & & .670 & & & & .834 \\
\hline$<$ Median & $4(14.2)$ & $6(21.4)$ & $9(32.2)$ & $9(32.2)$ & & $5(17.8)$ & $7(25.0)$ & $16(57.2)$ & \\
\hline$\geq$ Median & $4(14.2)$ & $9(32.2)$ & $7(25.0)$ & $8(28.6)$ & & $3(12.0)$ & $9(36.0)$ & $13(52.0)$ & \\
\hline Type of surgical resection & & & & & .266 & & & & .133 \\
\hline GTR & $3(15.8)$ & $4(21.1)$ & $6(31.6)$ & $6(31.6)$ & & $5(26.3)$ & $3(15.8)$ & $11(57.9)$ & \\
\hline STR & $5(20.0)$ & $7(28.0)$ & $7(28.0)$ & $6(24.0)$ & & $3(12.0)$ & $11(44.0)$ & $11(44.0)$ & \\
\hline Biopsy & 0 & $4(44.4)$ & 0 & $5(55.6)$ & & 0 & $2(22.0)$ & $29(54.7)$ & \\
\hline Ki LI (\%) & & & & & $<.001$ & & & & .005 \\
\hline$<14$ & $8(21.6)$ & $15(40.5)$ & $10(27.1)$ & $4(10.8)$ & & $8(21.6)$ & $14(37.8)$ & $15(40.6)$ & \\
\hline$\geq 14$ & 0 & 0 & $3(18.7)$ & $13(81.3)$ & & 0 & $2(12.5)$ & $14(87.5)$ & \\
\hline Therapeutic modalities & & & & & .030 & & & & .287 \\
\hline RTH only & 0 & $3(25.0)$ & $3(25.0)$ & $6(50.0)$ & & $1(8.3)$ & $2(16.7)$ & $9(75.0)$ & \\
\hline CCRT & $5(35.7)$ & $4(28.6)$ & 0 & $5(35.7)$ & & $4(28.6)$ & $3(21.4)$ & $7(50.0)$ & \\
\hline CCRT and adjuvant TMZ & $3(11.2)$ & $8(29.6)$ & $10(37.0)$ & $6(22.2)$ & & $3(11.1)$ & $11(40.7)$ & $13(48.2)$ & \\
\hline
\end{tabular}

Significant at $p<.05$.

ALK-1, anaplastic lymphoma kinase 1; h-TERT, human telomerase reverse transcriptase; IHC, immunohistochemistry; CC, corpus callosum; FP, fronto-parietal; PO, parieto-occipital; TO, tempero-occipital; PS, parasagittal; TP, tempero-parietal; GTR, gross total resection; STR, subtotal resection; Ki LI, Ki labeling index; RTH, radiotherapy; CCRT, concurrent chemoradiotherapy; TMZ, temozolomide.

aFisher exact test was used in this table except when $\leq 20 \%$ of the cells have expected count less than 5 chi-square test was used instead.

ALK-A was detected in four cases out of 17 IHC stains that were strongly positive for ALK-1 expression, and ALK-CNG was noted in 12 cases. This is consistent with two early studies done by Hudson et al. [18] and Kulig et al. [19], which found that $60 \%$ and $48.2 \%$ of their GBM cases possessed ALK gene gain/amplification by FISH, respectively. On the other hand, our results are contrary to those presented by Karagkounis et al. [5] and Chiba et al. [4] with respect to the $A L K$ gene via FISH analysis. Karagkounis et al. [5] revealed only one case of GBM showed ALK-A of the $2 \mathrm{p}$ domain. Likewise, Chiba et al. [4] revealed no amplification of the ALK locus.
A study on non-small lung cancer reported that $A L K$ gene nontranslocated cancers are commonly related to unstable chromosomes and ALK-CNG, while the ALK gene translocated cancers represent a low number of $A L K$ gene copies [20]. This may clarify our findings of the association between $A L K$ non-rearranged tumors with increased ALK gene copies (ALK-A and ALK-CG) and moderate to strong expression of ALK-I IHC, while the ALKrearranged tumors without $A L K$ gene extra-copies were associated with negative to weak ALK-I IHC expression. The relationship between ALK gene extra-copies (ALK-A and ALK-CG) by FISH and ALK protein expression is still debatable. On one hand, a study 
Table 3. Association between ALK gene alteration, clinicopathological parameters, and ALK-1 and h-TERT expression

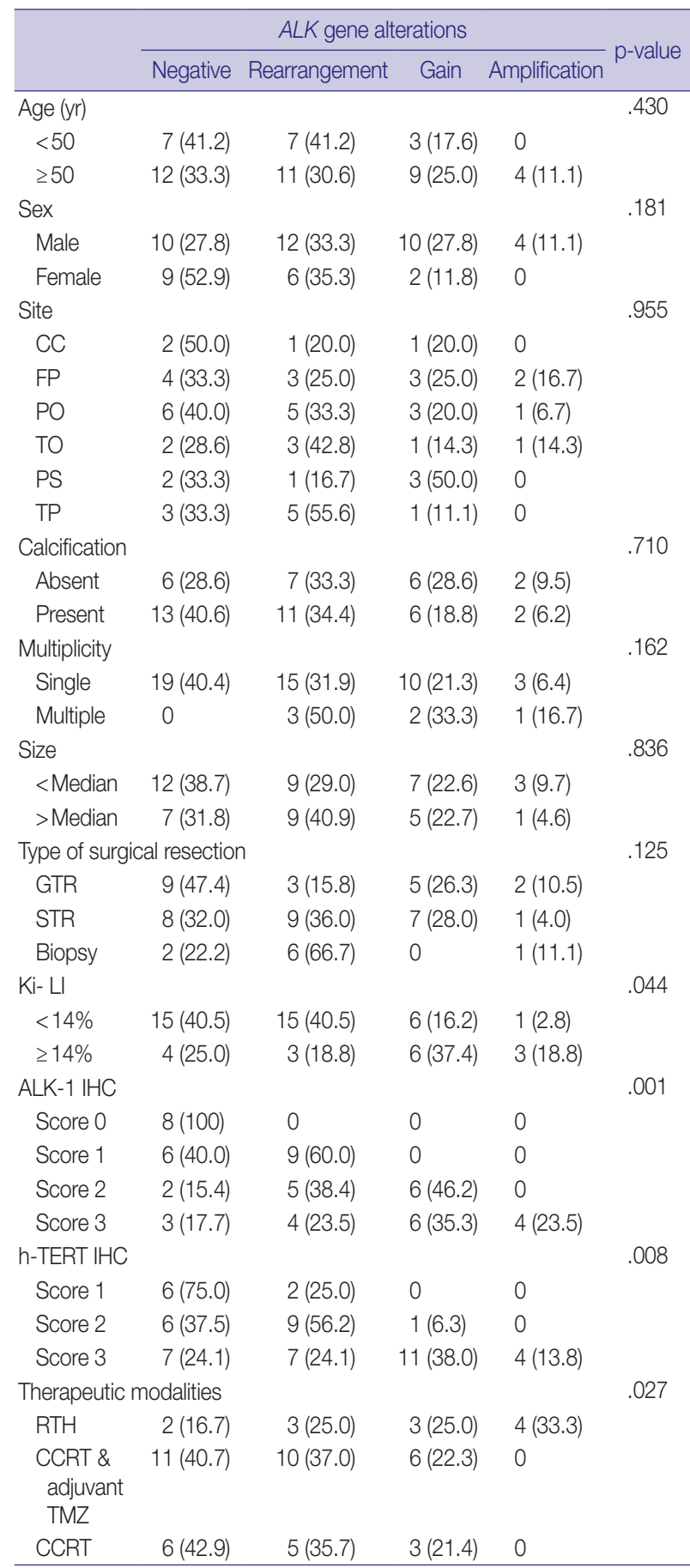

Significant at $p<.05$.

ALK-1, anaplastic lymphoma kinase 1 ; $\mathrm{h}$-TERT, human telomerase reverse transcriptase; CC, corpus callosum; FP, fronto-parietal; PO, parieto-occipital; TO, tempero-occipital; PS, parasagittal; TP, tempero-parietal; GTR, gross total resection; STR, subtotal resection; Ki LI, Ki labeling index; $I \mathrm{HC}$, immunohistochemistry; RTH, radiotherapy; CCRT, concurrent chemoradiotherapy; TMZ, temozolomide.
Table 4. Correlation between ALK gene alterations, ALK-1, and hTERT IHC protein expression

\begin{tabular}{lccc}
\hline & \multicolumn{3}{c}{ Spearman's rho } \\
\cline { 2 - 4 } & $\begin{array}{c}\text { h-TERT } \\
\text { expression }\end{array}$ & $\begin{array}{c}\text { ALK-1 } \\
\text { expression }\end{array}$ & $\begin{array}{c}\text { ALK gene } \\
\text { alterations }\end{array}$ \\
\hline h-TERT expression & & & \\
Correlation coefficient & 1.000 & 0.602 & 0.476 \\
Sig. (2-tailed) & & $0.002^{\mathrm{a}}$ & $0.007^{\mathrm{a}}$ \\
No. & 53 & 53 & 53 \\
ALK-1 expression & & & \\
Correlation coefficient & 0.602 & 1.000 & 0.616 \\
Sig. (2-tailed) & $0.002^{\mathrm{a}}$ & & $0.001^{\mathrm{a}}$ \\
$\quad$ No. & 53 & 53 & 53 \\
ALK gene alterations & & & \\
Correlation coefficient & 0.476 & 0.616 & 1.000 \\
Sig. (2-tailed) & $0.007^{\mathrm{a}}$ & $0.001^{\mathrm{a}}$ & \\
No. & 53 & 53 & 53 \\
\hline
\end{tabular}

ALK-1, anaplastic lymphoma kinase 1 ; h-TERT, human telomerase reverse transcriptase; $I \mathrm{HC}$, immunohistochemistry.

aSignificant; correlation is significant at the 0.01 level (2-tailed).

revealed that $A L K$ gene aberrations detected by FISH and ALK overexpression by IHC in rhabdomyosarcoma were significantly correlated [21]. On the other hand, other studies found no association between $A L K$ gene aberrations detected by FISH and ALK expression by IHC in various cancer types such as esophageal squamous cell carcinoma and colorectal carcinoma [22]. Furthermore, a study of a neuroblastoma cell line harboring ALK-A was accompanied by reduction of ALK levels as a result of Nlinked glycosylation inhibition with subsequent inhibition of its phosphorylated downstream molecules such as AKT and STAT3 [23]. This controversy between the relationship between $A L K$ gene extra-copies on FISH and ALK protein expression may be attributed to either transcriptional or post-transcriptional modifications, or degradation, such as $\mathrm{N}$-linked glycosylation activation or inhibition, which lead to $A L K$ activation and reduction, respectively.

Regarding h-TERT immunohistochemical expression, the current work revealed strong h-TERT nuclear expression in about half of the studied cases; this is compatible with the study done by Potharaju et al. [10], which showed that $60 \%$ of GBM patients expressed strong h-TERT. In spite of the use of h-TERT IHC as a mirror to detect tumors harboring TERT-mutation, h-TERT IHC was not able to identify the differences between TERTmutated GBMs and TERT-nonmutant GBMs. h-TERT protein overexpression was noted even among gliomas with wildtype TERT. Moreover, h-TERT IHC widely varied through the TERTmutant gliomas such as oligodendrogliomas and GBMs. This indicates that h-TERT IHC expression may be regulated by various mechanisms along with TERT promoter mutations [24]. 


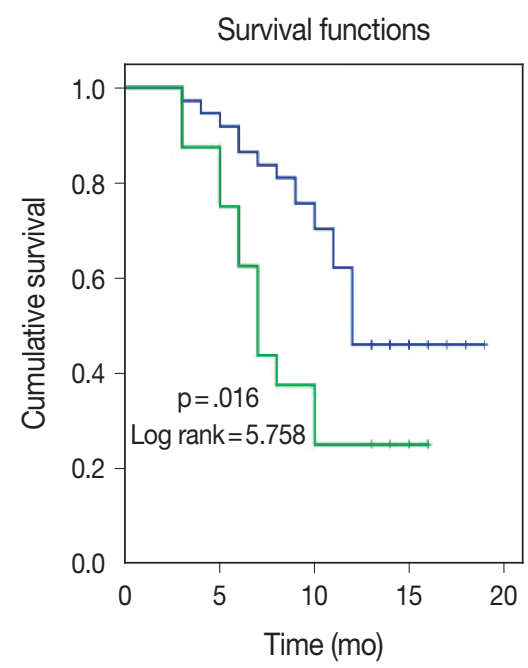

$\begin{aligned} & \text { Ki LI } \\ \neg & <14 \% \\ \neg & \geq 14 \% \\ + & <14 \% \text {-censored } \\ + & \geq 14 \% \text {-censored }\end{aligned}$
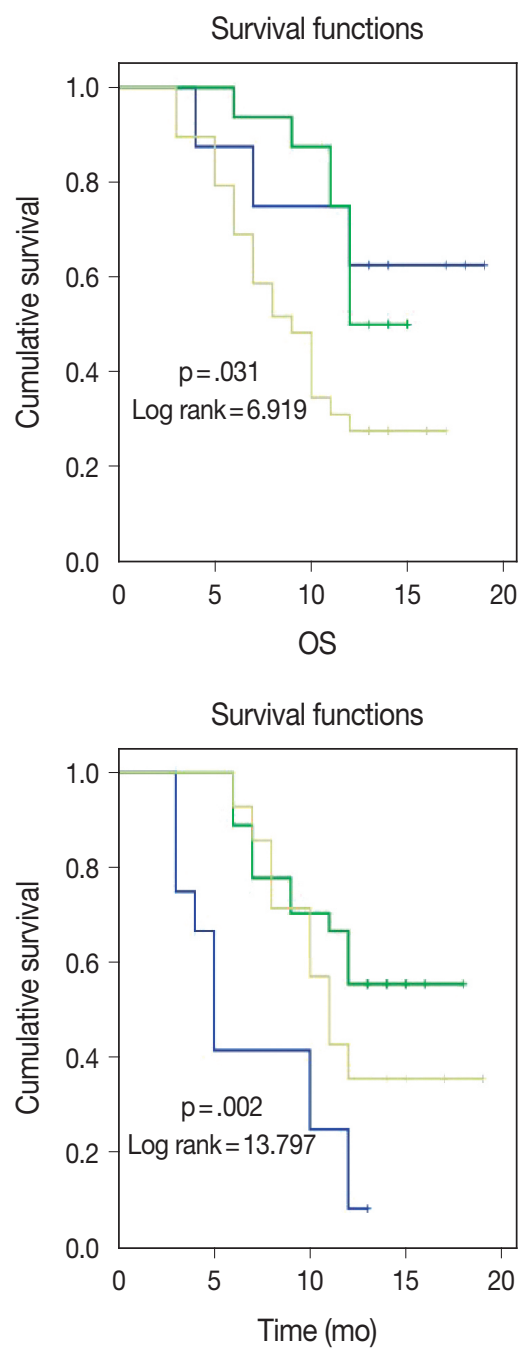
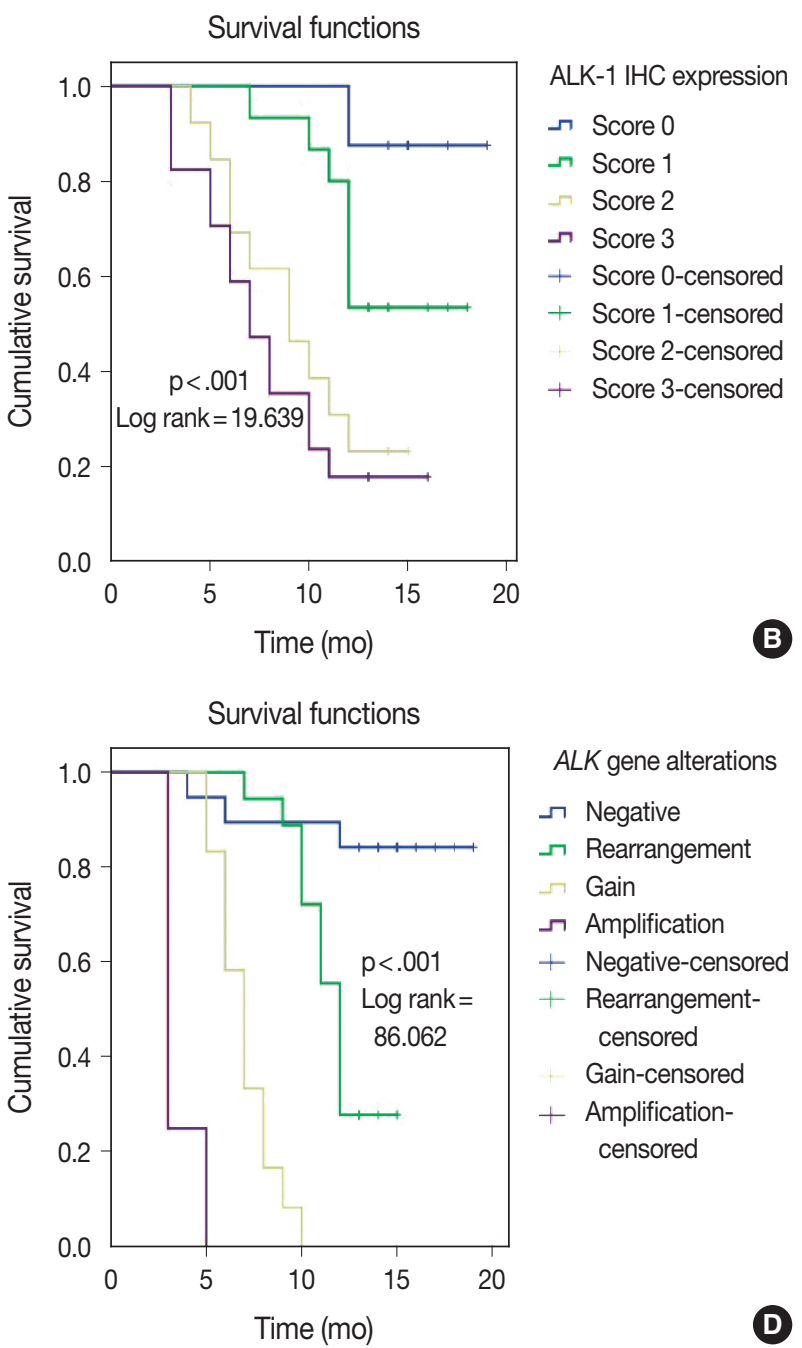

Fig. 3. Overall survival (OS) for anaplastic lymphoma kinase 1 (ALK-1), human telomerase reverse transcriptase (h-TERT) immunohistochemistry $(\mathrm{HC})$ expression, and ALK gene alterations. (A) High Ki labeling index (Ki LI) is associated with poor OS. (B) ALK-1 score 3 is associated with poor OS. (C) TERT score 3 is associated with poor OS. (D) ALK gene amplification is associated with poor OS. (E) Patients treated with adjuvant radiotherapy only had poor OS compared to those who were treated with concurrent chemoradiotherapy (CCRT) or CCRT and adjuvant temozolomide (TMZ). 

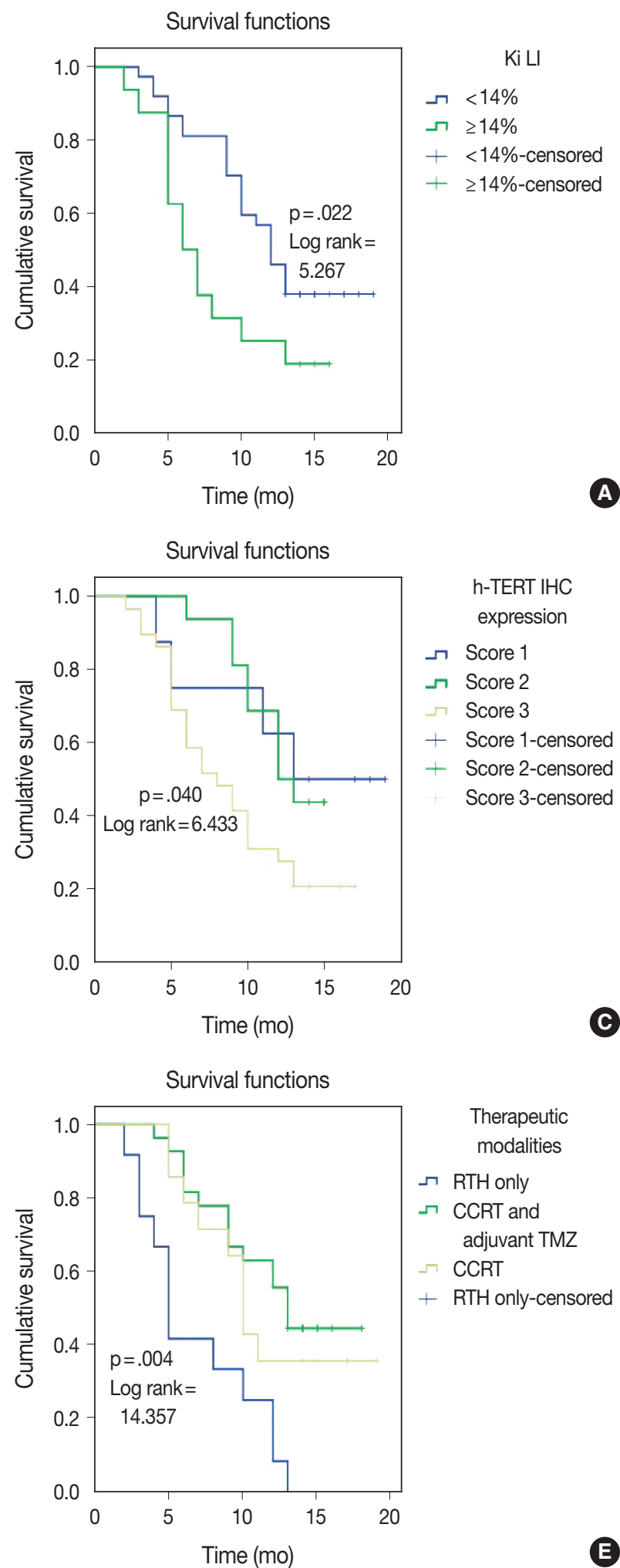
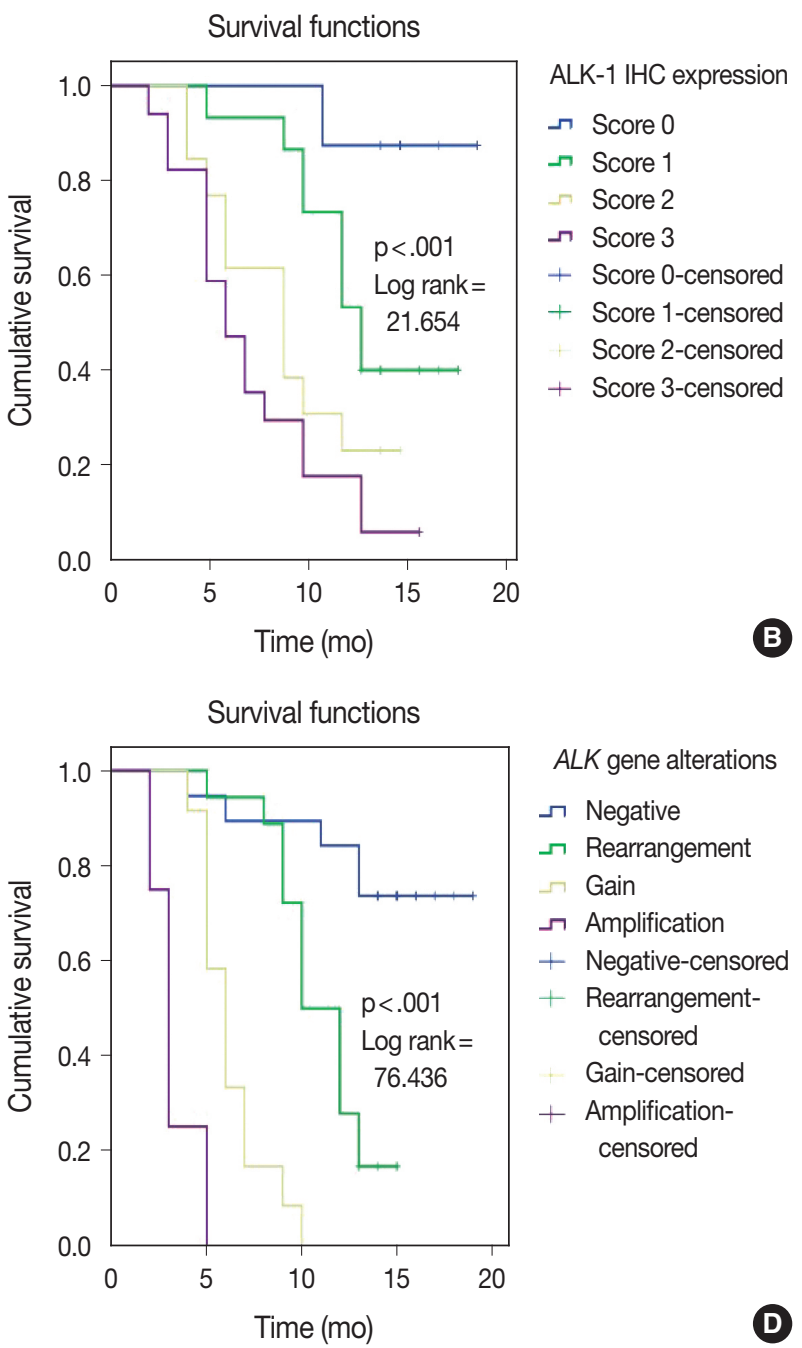

Fig. 4. Progression-free survival (PFS) for anaplastic lymphoma kinase 1 (ALK-1), human telomerase reverse transcriptase (h-TERT) immunohistochemistry $(\mathrm{HC})$ expression, and ALK gene alterations. (A) High Ki labeling index (Ki LI) is associated with short PFS. (B) ALK-1 score 3 is associated with poor PFS. (C) TERT score 3 is associated with poor PFS. (D) ALK gene amplification is associated with poor PFS. (E) Patients treated with adjuvant radiotherapy only had poor PFS compared to those who were treated with concurrent chemoradiotherapy (CCRT) or CCRT and adjuvant temozolomide (TMZ). 
Table 5. Kaplan-Meier analysis for OS and PFS

\begin{tabular}{|c|c|c|c|c|}
\hline \multirow{2}{*}{ Parameter } & \multicolumn{2}{|l|}{ OS } & \multicolumn{2}{|l|}{ PFS } \\
\hline & Log-rank (chi-square) & $p$-value & Log-rank (chi-square) & $p$-value \\
\hline Age (<50 yr vs. $\geq 50$ yr) & 3.146 & .076 & 2.419 & .120 \\
\hline Sex (male vs. female) & 3.326 & .119 & 3.887 & .153 \\
\hline Site (CC vs. FP vs. PO vs. TO vs. PS vs. TP) & 3.398 & .639 & 4.216 & .519 \\
\hline Calcification (absent vs. present) & 0.666 & .414 & 0.253 & .615 \\
\hline Multiplicity (single vs. multiple) & 3.993 & .254 & 3.505 & .190 \\
\hline Size (<median vs. > median) & 0.010 & .921 & 0.048 & .827 \\
\hline Type of surgical resection (GTR vs. STR vs. biopsy) & 0.066 & .967 & 0.168 & .919 \\
\hline Ki LI (<14\% vs. $\geq 14 \%)$ & 5.758 & .016 & 5.267 & .022 \\
\hline ALK-1 IHC (score 0 vs. score 1 vs. score 2 vs. score 3 ) & 19.639 & $<.001$ & 21.654 & $<.001$ \\
\hline h-TERT IHC (score 1 vs. score 2 vs. score 3) & 6.919 & .031 & 6.433 & .040 \\
\hline ALK gene alterations (negative rearrangement vs. gain amplification) & 86.062 & $<.001$ & 76.436 & $<.001$ \\
\hline Therapeutic modalities (RTH only vs. CCRT vs. CCRT and adjuvant TMZ) & 13.797 & .002 & 14.357 & .004 \\
\hline
\end{tabular}

Significant at $p<0.5$.

OS, overall survival; PFS, progression-free survival; CC, corpus callosum; FP, fronto-parietal; PO, parieto-occipital; TO, tempro-occipital; PS, parasagittal; TP, tempro-parietal; GTR, gross total resection; STR, subtotal resection; Ki LI, Ki labeling index; ALK-1, anaplastic lymphoma kinase 1; IHC, immunohistochemistry; h-TERT, human telomerase reverse transcriptase; RTH, radiotherapy; CCRT, concurrent chemoradiotherapy; TMZ, temozolomide.

Table 6. Multivariate analysis for significant predictors of OS and PFS

\begin{tabular}{|c|c|c|c|c|c|c|c|c|c|c|}
\hline & \multicolumn{5}{|c|}{ OS } & \multicolumn{5}{|c|}{ PFS } \\
\hline & B & SE & $p$-value & $\mathrm{HR}$ & $95 \%$ Cl for $\operatorname{Exp}(\mathrm{B})$ & B & SE & $p$-value & $\mathrm{HR}$ & $95 \%$ Cl for $\operatorname{Exp}(B)$ \\
\hline Ki LI & 0.125 & 0.468 & .790 & 1.133 & $0.453-2.833$ & -0.001 & 0.435 & .998 & 0.099 & $0.426-2.344$ \\
\hline ALK-1 protein expression & 0.357 & 0.347 & .304 & 0.429 & $0.724-2.821$ & 0.505 & 0.306 & .099 & 1.657 & $0.909-3.010$ \\
\hline h-TERT protein expression & -0.368 & 0.411 & .371 & 0.692 & $0.310-1.549$ & -0.352 & 0.356 & .322 & 0.703 & $0.350-1.411$ \\
\hline ALK gene alterations & 2.017 & 0.421 & $<.001$ & 7.514 & $3.292-17.155$ & 1.550 & 0.338 & $<.001$ & 4.711 & $2.429-9.136$ \\
\hline Therapeutic modalities & 0.050 & 0.311 & .872 & 1.052 & $0.571-1.935$ & -0.007 & 0.295 & .982 & 0.993 & $0.558-1.769$ \\
\hline
\end{tabular}

Significant at $\mathrm{p}<0.5$.

OS, overall survival; PFS, progression-free survival; HR, hazards ratio; Cl, confident interval; Ki LI, Ki labeling index; ALK-1, anaplastic lymphoma kinase 1; hTERT, human telomerase reverse transcriptase.

An interesting finding is the presence of a significant association between h-TERT expression and the presence of tumor calcification in the present study, which may be attributed to the hypoxic state in GBM that leads to activation of hypoxia-inducible factor- $1 \alpha(\mathrm{HIF}-1 \alpha)$ in response to hypoxic status with subsequent increase in intracellular calcium $\left(\mathrm{Ca}^{2+}\right)$ and promotion of the $\mathrm{Ca}^{2+}$ signaling pathway [25]. Furthermore, activation of HIF-1 $\alpha$ enhances h-TERT transcription [26].

Concerning the relationship between Ki LI and ALK-1/hTERT protein expression, all the aforementioned biomarkers had a reportedly positive association with Ki LI [4]. Persson and Englund [11] discerned that high values of Ki LI were not significantly associated with high h-TERT staining. The current study showed that high Ki LI had a poor impact on OS and PFS. This is in agreement with Persson and Englund [11], and in contrast with other studies, which noted no effect of Ki LI on OS $[11,27,28]$. The prognostic value of Ki LI in GBM is still uncertain, as the distribution of proliferative index was different in variable areas within the same tumor and different cutoff points are used in the literature.

Overall, there was a strong positive correlation between ALK-1 and h-TERT IHC protein expression in the current research. All GBM cases displayed necrosis and/or microvascular proliferation, which is essential for their diagnosis [29]. These findings were corresponding to a hypoxic state of the microenvironment. ALK expression was significantly higher in tumor cells in hypervascular lesions as compared to those adjacent to necrotic foci in GBMs, and was positively correlated with the microvascular density as determined by CD34 expression. Overexpression of ALK induced an enhancement of the HIF-1 $\alpha /$ vascular endothelial growth factor-A axis through activation of Stat3 [4]. Furthermore, a study done by Marzec et al. [30] reported that ALKpositive T-cell lymphoma expresses HIF1 $\alpha$. HIF1 $\alpha$ mRNA expression is induced in ALK-positive T-cell lymphoma by the transcription of nucleophosmin/ALK tyrosine kinase. NPM/ ALK activates the HIF1 $\alpha$ gene through the STAT3 transcription factor [30].

GBMs contain considerable hypoxic areas within the tumor. 
Potharaju et al. [10] suggested that an intratumoral decrease in oxygen concentration can augment HIF-1 $\alpha$ expression and enhance h-TERT transcription and telomerase, which in turn lead to proliferation of cancer stem cells [26]. Consequently, both ALK-1 and h-TERT expression are highly expressed in the hypervascular area of GBM, depending on HIF-1 $\alpha$ activation. This may explain the close relationship between ALK and h-TERT expression in GBM.

Regarding OS, high ALK-1 expression, high h-TERT expression, and ALK-A were associated with poor OS in the studied cases. This result was in congruent with a study [4] showing that loss of ALK expression had improved OS in contrast to the ALKpositive cases and another report [10] showing that patients with absent or weak h-TERT expression had significantly longer OS than patients with strong h-TERT expression. However, the current findings are inconsistent with those of Karagkounis et al. [5] and Persson and Englund [11], which noted no significant impact of ALK-1 and h-TERT expression on OS, respectively.

Interestingly, we found that $A L K$ gene alteration was an independent prognostic factor for OS. This in agreement with a previous observation, which revealed that patients who had ALKA died within one month from diagnosis [5].

In the current study, the type of therapeutic modality was positively associated with both ALK-1 protein expression and ALK gene alterations. Patients who were treated with adjuvant radiotherapy only had poor survival outcomes compared to those who were treated with CCRT or CCRT and maintenance TMZ. The best survival outcome was among patients who were treated with adjuvant CCRT and maintenance TMZ. A potential therapeutic application in patients harboring ALK-A was noted in in vitro studies that applied specific ALK inhibitors to cell lines with ALK-A [7]. Furthermore, Le Rhun et al. [13] suggested that GBM patients with ALK protein expression and ALK-CNG could benefit from novel targeted ALK inhibitors (crizotinib). Therefore, ALK-targeting therapy may be used for treatment of GBM patients with $A L K$ gene alterations, taking into consideration the h-TERT mutation.

The limitation of this research is that it lacks application of IDH-1 IHC for subdivision of GBM into molecular subtypes according to IDH status and for correlation with ALK-1 and hTERT IHC, which will add to the research. This point is recommended in future studies.

In summary, Break-Apart ALK FISH is a reliable diagnostic technique that can be applied with ease on FFPE tissue whenever the exact fusion partners are indefinite. Furthermore, ALK gene alterations have a significant prognostic impact on GBM patients. Subsequent studies with a larger number of GBM cases are recommended for better evaluation of the role of ALK-1, hTERT, and $A L K$ gene alterations in tumor progression and related mechanisms.

The current work concluded that high protein expression of ALK-1, h-TERT, and ALK-A had poor impact on the prognosis of GBM. Accordingly, ALK-1 protein expression, h-TERT protein expression, and $A L K$ gene alteration detection could be used as valuable prognostic markers in GBM patients. The type of therapeutic modality was positively associated with $A L K$ gene alterations. The use of ALK-targeting therapy as part of treatment plan for GBM patients with $A L K$ gene alterations is the goal, and requires further studies with a larger sample size.

\section{Ethics Statement}

The research was approved by the Committee of Medical Ethics, Faculty of Medicine, Assiut University IRB. No. 17300482. A written informed consent for participation and publication was obtained from each participant after receiving information about the details of the study. Confidentiality of patients' records was assured and maintained throughout the study.

\section{Availability of Data and Material}

The datasets generated or analyzed during the current study are available from the corresponding author on reasonable request.

\section{Code Availability}

Not applicable.

\section{ORCID}

Marwa T. Hussien https://orcid.org/0000-0001-8561-8501

\section{Author Contributions}

Conceptualization: DE, MTH. Data curation: DE, MTH, DFT. Formal analysis: DE, MTH. Methodology: DE, MTH, DFT. Resources: DE, MTH, DFT. Writing—original draft: DE, MTH. Writing—review \& editing: AMA, AH. Approval of final manuscript: all authors.

\section{Conflicts of Interest}

The authors declare that they have no potential conflicts of interest.

\section{Funding Statement}

No funding to declare.

\section{References}

1. Stoyanov GS, Dzhenkov D, Ghenev P, Iliev B, Enchev Y, Tonchev AB. Cell biology of glioblastoma multiforme: from basic science to diagnosis and treatment. Med Oncol 2018; 35: 27.

2. Louis DN, Perry A, Reifenberger G, et al. The 2016 World Health Organization classification of tumors of the central nervous system: a summary. Acta Neuropathol 2016; 131: 803-20.

3. Chiarle R, Voena C, Ambrogio C, Piva R, Inghirami G. The anaplastic lymphoma kinase in the pathogenesis of cancer. Nat Rev Cancer 2008; 8: 11-23.

4. Chiba R, Akiya M, Hashimura M, et al. ALK signaling cascade con- 
fers multiple advantages to glioblastoma cells through neovascularization and cell proliferation. PLoS One 2017; 12: e0183516.

5. Karagkounis G, Stranjalis G, Argyrakos T, et al. Anaplastic lymphoma kinase expression and gene alterations in glioblastoma: correlations with clinical outcome. J Clin Pathol 2017; 70: 593-9.

6. Wojas-Krawczyk K, Krawczyk PA, Ramlau RA, et al. The analysis of $A L K$ gene rearrangement by fluorescence in situ hybridization in non-small cell lung cancer patients. Contemp Oncol (Pozn) 2013; 17: 484-92.

7. Zito Marino F, Botti G, Aquino G, et al. Unproductive effects of $A L K$ gene amplification and copy number gain in non-small-cell lung cancer: $A L K$ gene amplification and copy gain in NSCLC. Int J Mol Sci 2020; 21: 4927.

8. Hafezi F, Perez Bercoff D. The solo play of TERT promoter mutations. Cells 2020; 9: 749.

9. Leao R, Apolonio JD, Lee D, Figueiredo A, Tabori U, Castelo-Branco P. Mechanisms of human telomerase reverse transcriptase (hTERT) regulation: clinical impacts in cancer. J Biomed Sci 2018; 25: 22.

10. Potharaju M, Mathavan A, Mangaleswaran B, et al. Clinicopathological analysis of HIF-1alpha and TERT on survival outcome in glioblastoma patients: a prospective, single institution study. J Cancer 2019; 10: 2397-406.

11. Persson A, Englund E. Different assessments of immunohistochemically stained Ki-67 and hTERT in glioblastoma multiforme yield variable results: a study with reference to survival prognosis. Clin Neuropathol 2008; 27: 224-33.

12. Stupp R, Mason WP, van den Bent MJ, et al. Radiotherapy plus concomitant and adjuvant temozolomide for glioblastoma. N Engl J Med 2005; 352: 987-96.

13. Le Rhun E, Chamberlain MC, Zairi F, et al. Patterns of response to crizotinib in recurrent glioblastoma according to ALK and MET molecular profile in two patients. CNS Oncol 2015; 4: 381-6.

14. Alidousty C, Duerbaum N, Wagener-Ryczek S, et al. Prevalence and potential biological role of TERT amplifications in ALK translocated adenocarcinoma of the lung. Histopathology 2021; 78: 57885.

15. Saha R, Chatterjee U, Mandal S, Saha K, Chatterjee S, Ghosh SN. Expression of phosphatase and tensin homolog, epidermal growth factor receptor, and $\mathrm{Ki}-67$ in astrocytoma: a prospective study in a tertiary care hospital. Indian J Med Paediatr Oncol 2014; 35: 14955.

16. McLeer-Florin A, Moro-Sibilot D, Melis A, et al. Dual IHC and FISH testing for $A L K$ gene rearrangement in lung adenocarcinomas in a routine practice: a French study. J Thorac Oncol 2012; 7: 348-54.
17. Salido M, Pijuan L, Martinez-Aviles L, et al. Increased ALK gene copy number and amplification are frequent in non-small cell lung cancer. J Thorac Oncol 2011; 6: 21-7.

18. Hudson L, Kulig K, Young D, McLendon R, Abemethy A. ALK and cMET expression in glioblastoma multiforme: implications for therapeutic targeting. Mol Cancer Ther 2011; 10(11 Suppl): A42.

19. Kulig K, McLendon RE, Locke SC, et al. MET and ALK in glioblastoma multiforme (GBM): comparison of IHC and FISH. J Clin Oncol 2012; 30(15 Suppl): 2021.

20. Peretti U, Ferrara R, Pilotto S, et al. ALK gene copy number gains in non-small-cell lung cancer: prognostic impact and clinico-pathological correlations. Respir Res 2016; 17: 105.

21. Lee JS, Lim SM, Rha SY, et al. Prognostic implications of anaplastic lymphoma kinase gene aberrations in rhabdomyosarcoma; an immunohistochemical and fluorescence in situ hybridisation study. J Clin Pathol 2014; 67: 33-9.

22. Schoppmann SF, Streubel B, Birner P. Amplification but not translocation of anaplastic lymphoma kinase is a frequent event in oesophageal cancer. Eur J Cancer 2013; 49: 1876-81.

23. Del Grosso F, De Mariano M, Passoni L, Luksch R, Tonini GP, Longo L. Inhibition of N-linked glycosylation impairs ALK phosphorylation and disrupts pro-survival signaling in neuroblastoma cell lines. BMC Cancer 2011; 11: 525.

24. Masui K, Komori T, Kato Y, et al. Elevated TERT expression in TERT-wildtype adult diffuse gliomas: histological evaluation with a novel TERT-specific antibody. Biomed Res Int 2018; 2018: 7945845.

25. Leclerc C, Haeich J, Aulestia FJ, et al. Calcium signaling orchestrates glioblastoma development: facts and conjunctures. Biochim Biophys Acta 2016; 1863: 1447-59.

26. Nishi H, Nakada T, Kyo S, Inoue M, Shay JW, Isaka K. Hypoxia-inducible factor 1 mediates upregulation of telomerase (hTERT). Mol Cell Biol 2004; 24: 6076-83.

27. Alkhaibary A, Alassiri AH, AlSufiani F, Alharbi MA. Ki-67 labeling index in glioblastoma; does it really matter? Hematol Oncol Stem Cell Ther 2019; 12: 82-8.

28. Tsidulko AY, Kazanskaya GM, Kostromskaya DV, et al. Prognostic relevance of NG2/CSPG4, CD44 and Ki-67 in patients with glioblastoma. Tumour Biol 2017; 39: 1010428317724282.

29. Abdelzaher E. Glioblastoma multiforme, NOS [Internet]. Bingham Farms: PathologyOutlines.com, 2020 [cited 2020 May 27]. Available from: https://www.pathologyoutlines.com/topic/cnstumorglioblastomagiantcell.html.

30. Marzec M, Liu X, Wong W, et al. Oncogenic kinase NPM/ALK induces expression of HIFlalpha mRNA. Oncogene 2011; 30: 1372-8. 